OPEN ACCESS

Edited by:

Aixin Yan,

University of Hong Kong, Hong Kong

Reviewed by:

Atte Von Wright,

University of Eastern Finland, Finland

Fangyou Yu,

First Affiliated Hospital of Wenzhou

Medical University, China

*Correspondence:

Malcolm J. Horsburgh

m.j.horsburgh@liverpool.ac.uk

Specialty section:

This article was submitted to

Antimicrobials, Resistance

and Chemotherapy,

a section of the journal

Frontiers in Microbiology

Received: 28 July 2016 Accepted: 06 January 2017

Published: 25 January 2017

Citation:

Moran JC, Alorabi JA and Horsburgh MJ (2017) Comparative

Transcriptomics Reveals Discrete

Survival Responses of S. aureus and S. epidermidis to Sapienic Acid.

Front. Microbiol. 8:33.

doi: 10.3389/fmicb.2017.00033

\section{Comparative Transcriptomics Reveals Discrete Survival Responses of $S$. aureus and S. epidermidis to Sapienic Acid}

\author{
Josephine C. Moran, Jamal A. Alorabi and Malcolm J. Horsburgh* \\ Functional and Comparative Genomics, Institute of Integrative Biology, University of Liverpool, Liverpool, UK
}

Staphylococcal colonization of human skin is ubiquitous, with particular species more frequent at different body sites. Whereas Staphylococcus epidermidis can be isolated from the skin of every individual tested, Staphylococcus aureus is isolated from $<5 \%$ of healthy individuals. The factors that drive staphylococcal speciation and niche selection on skin are incompletely defined. Here we show that $S$. aureus is inhibited to a greater extent than S. epidermidis by the sebaceous lipid sapienic acid, supporting a role for this skin antimicrobial in selection of skin staphylococci. We used RNA-Seq and comparative transcriptomics to identify the sapienic acid survival responses of $S$. aureus and $S$. epidermidis. Consistent with the membrane depolarization mode of action of sapienic acid, both species shared a common transcriptional response to counteract disruption of metabolism and transport. The species differed in their regulation of SaeRS and VraRS regulons. While S. aureus upregulated urease operon transcription, S. epidermidis upregulated arginine deiminase, the oxygen-responsive NreABC nitrogen regulation system and the nitrate and nitrite reduction pathways. The role of $S$. aureus ACME and chromosomal arginine deiminase pathways in sapienic acid resistance was determined through mutational studies. We speculate that ammonia production could contribute to sapienic acid resistance in staphylococci.

Keywords: RNA-Seq, Staphylococcus aureus, Staphylococcus epidermidis, sapienic acid, fatty acid, skin, colonization

\section{INTRODUCTION}

Staphylococci are major commensal colonizers of healthy human skin and leading causes of hospital-acquired infections, responsible predominantly for wound and device-associated infections. Understanding skin survival mechanisms of staphylococci is vital, as individuals colonized by Staphylococcus aureus are at greater risk of infections during hospitalization (Kluytmans et al., 1997; Davis et al., 2004).

Unlike those coagulase-negative staphylococci that are skin-dwelling, the primary human niche of coagulase-positive S. aureus is the nares, with skin colonization being transient and seeded from this location (Moss and Squire, 1948; Kluytmans and Wertheim, 2005; Cho et al., 2010). A comparison of responses and resistance mechanisms between $S$. aureus and these closely related, long-term skin colonizers, such as $S$. epidermidis, therefore provides a useful tool to 
investigate functionalities required for skin colonization and persistence (Coates et al., 2014). Such investigations have increasing relevance with the emergence of community-acquired MRSA lineages, such as USA300 which cause increased skin pathology (Moran et al., 2006; Li et al., 2009).

Atopic dermatitis is a disease presenting as dry, flaky skin lesions, abscesses, and unusually high levels of $S$. aureus skin colonization (Higaki et al., 1999; Bieber, 2008; Kong et al., 2012). Many host factors of skin are altered in atopic dermatitis, including levels of antimicrobial peptides, antimicrobial fatty acids, and sphingosines, all of which have been associated with $S$. aureus exclusion (Schafer and Kragballe, 1991; Arikawa et al., 2002; Cho et al., 2010). Levels of sapienic acid in particular were determined to be inversely proportional to levels of S. aureus (Takigawa et al., 2005), identifying sapienic acid as a strong candidate host factor that contributes to prevention of long-term skin colonization by S. aureus.

Recent studies revealed the effects that sapienic acid and other skin fatty acids have on S. aureus survival (Kenny et al., 2009; Cartron et al., 2014; Neumann et al., 2015). Together these studies showed that unsaturated long-chain fatty acids, including sapienic and linoleic acids, cause membrane depolarization in S. aureus leading to large transcriptional changes, especially those pathways associated with cellular energetics (Kenny et al., 2009; Neumann et al., 2015). From the transcriptomic response, it is inferred that the membrane depolarisation leads to disruption of the electron transport chain (Kenny et al., 2009; Neumann et al., 2015).

Here we show that the mean sapienic acid MIC of S. epidermidis strains is greater than S. aureus. Consequently, RNA-Seq was used to compare sapienic acid transcriptional responses with the aim of highlighting skin survival determinants. These investigations form the basis to determine whether sapienic acid responses discriminate staphylococcal species based on their skin-dwelling propensity.

\section{MATERIALS AND METHODS}

\section{Bacterial Strains and Culture}

Strains used in this study are listed in Table 1. Overnight cultures were grown for $18 \mathrm{~h}$ at $37^{\circ} \mathrm{C}$ with shaking. Todd Hewitt broth (THB) or agar (THA) was used as the culture media for all experiments. Sapienic acid (Matreya) stock solution was prepared at $8 \mathrm{mg} \mathrm{ml}^{-1}$ in ethanol. Antibiotics were incorporated at concentrations of $12.5 \mu \mathrm{g} \mathrm{ml}^{-1}$ tetracycline, $100 \mu \mathrm{g} \mathrm{ml}^{-1}$ ampicillin, $10 \mu \mathrm{g} \mathrm{ml}^{-1}$ chloramphenicol, and $5 \mu \mathrm{g} \mathrm{ml} \mathrm{m}^{-1}$ erythromycin, when appropriate.

\section{Minimum Inhibitory Concentration Assay}

Minimum inhibitory concentration (MIC) assays were performed using a broth microdilution method in 96 well plates, with final well volumes of $200 \mu \mathrm{l}$ and a sapienic acid concentration range of $200-0.8 \mu \mathrm{g} \mathrm{ml}^{-1}$. An inoculum of $\sim 10^{4}$ $\mathrm{CFU} \mathrm{ml} \mathrm{m}^{-1}$ was used.

\section{Growth and Sapienic Acid Challenge}

Overnight broth cultures were adjusted to an $\mathrm{OD}_{600}$ of 0.5 then diluted 25-fold in fresh medium prior to incubation in a water bath with shaking $(250 \mathrm{rpm})$ at $37^{\circ} \mathrm{C}$. Sapienic acid/ethanol was added to cell cultures in mid-exponential phase $\left(\mathrm{OD}_{600}\right.$ $\sim 0.5$ ) with equivalent volumes of ethanol added to control cultures. For RNA-Seq experiments, cells were harvested by centrifugation 20 min after challenge and suspended in RNAlater (Qiagen).

\section{RNA Extraction and Library Preparation}

For cell lysis, bacteria were pelleted at 6,000 RCF for $5 \mathrm{~min}$ at $4^{\circ} \mathrm{C}$ and suspended in $100 \mu \mathrm{TE}$ containing $6 \mathrm{mg} \mathrm{ml}^{-1}$ lysostaphin and $400 \mathrm{U} \mathrm{ml}^{-1}$ mutanolysin. Lysis was performed for $15 \mathrm{~min}$ at $37^{\circ} \mathrm{C}$ for $S$. aureus and $30 \mathrm{~min}$ for S. epidermidis. Subsequently, samples were treated with $25 \mu \mathrm{l}$ of proteinase $\mathrm{K}$ (Qiagen) for $30 \mathrm{~min}$ at $37^{\circ} \mathrm{C}$. RNA was extracted using the RNeasy kit (Qiagen). Samples were DNase-treated using turbo DNase (Ambion), and the DNase removed using the RNeasy MinElute clean up kit (Qiagen).

Depletion of rRNA was achieved with a Ribo-Zero magnetic kit for Gram-positive bacteria (Epicentre). The concentration of RNA was normalized before library construction using strand specific ScriptSeq kits (Epicentre); libraries were prepared by the Centre for Genomic Research (CGR), Liverpool. RNA-Seq samples were sequenced by paired-end sequencing using the HiSeq platform (Illumina).

\section{RNA-Seq Differential Expression Analysis}

Bowtie (Langmead et al., 2009) and Edge R (Robinson and Oshlack, 2010; Robinson et al., 2010) were used to map reads and determine the differentially expressed (DE) genes, respectively. Genes with mapped transcripts that had a false discovery rate $<0.05$, as determined by Benjamin and Hochberg analysis, were considered differentially expressed between control and test conditions.

Gene expression changes in biosynthetic pathways were associated using KEGG mapper-search and color (Kanehisa and Goto, 2000; Kanehisa et al., 2012). The S. aureus transcriptome meta-database (SATMD) (Nagarajan and Elasri, 2007) was used to compare sapienic acid DE gene sets with existing $S$. aureus DE gene sets.

\section{cDNA Generation and qPCR}

The tetro cDNA synthesis kit (Bioline) was used for cDNA synthesis using random hexamer primers and $2 \mu \mathrm{g}$ RNA per reaction. Table 2 lists the qPCR primers. Novel primers were designed using primer-BLAST (Ye et al., 2012). Primer efficiency for all primers was confirmed to be within $90-100 \%$ as described previously (Nolan et al., 2006). All qPCR reactions were performed using SensiFAST SYBR Hi-ROX kit (Bioline) with the ABI StepOnePlus (Life Technologies); data analysis used the ABI StepOnePlus software. At least two technical replicates and three biological replicates were used to determine fold change in gene expression between samples. 
TABLE 1 | Strains used in this study.

\begin{tabular}{|c|c|c|c|}
\hline Species & Strain & Description & Reference \\
\hline \multirow[t]{7}{*}{ S. epidermidis } & Rp62a & Intravascular catheter isolate & Christensen et al., 1982, 1985 \\
\hline & Tü3298 & Epidermin producer & Allgaier et al., 1986 \\
\hline & NCTC 1457 & PIA producer & Mack et al., 1992 \\
\hline & A19 & Recent skin (forearm) isolate & Kelly, 2013 \\
\hline & B19 & Recent skin (forearm) isolate & Kelly, 2013 \\
\hline & 016 & Recent skin (forearm) isolate & Kelly, 2013 \\
\hline & BL115 & Recent nasal isolate & Libberton, 2011 \\
\hline \multirow[t]{20}{*}{ S. aureus } & Newman & Osteomyelitis isolate & Duthie and Lorenz, 1952 \\
\hline & SH1000 & Lab strain (rsbU repaired 8325-4 derivative) & Horsburgh et al., 2002 \\
\hline & MSSA476 & Osteomyelitis isolate & Holden et al., 2004 \\
\hline & MRSA252 & Fatal bacteraemia isolate, MRSA & Holden et al., 2004 \\
\hline & BL014 & Recent nasal isolate & Libberton, 2011 \\
\hline & BL032 & Recent nasal isolate & Libberton, 2011 \\
\hline & SF8300 & CA-MRSA & Diep et al., 2008 \\
\hline & SF8300ax & SF8300 with ACME deletion & Diep et al., 2008 \\
\hline & Liv1245 & Newman arcA::tet from Liv692 & This study \\
\hline & Liv1247 & SF8300 arcA::tet from Liv692 & This study \\
\hline & Liv1249 & SF8300ax arcA::tet from Liv692 & This study \\
\hline & Liv692 & S. aureus SH1000 arcA::tet & Kenny et al., 2009 \\
\hline & Newman tagO & tagO::ery from SA113 tagO & This study \\
\hline & SA113 tagO & tagO::Ery & Bera et al., 2005 \\
\hline & Newman mcrA & NWMN_0050:: Ery & This study \\
\hline & Newman mcrA pSK5632 & Newman mcrA containing pSK5632 + mcrA & This study \\
\hline & Liv1023 & $m t \mid D:: t e t(\mathrm{SH} 1000)$ & Kenny et al., 2013 \\
\hline & Liv1024 & $m t \mid A B C D::$ tet $(\mathrm{SH} 1000)$ & Kenny et al., 2013 \\
\hline & RN4220 & Restriction deficient strain & Kreiswirth et al., 1983 \\
\hline & $\mathrm{SH} 1000 \mathrm{mnhF}$ & $m n h F$ in frame unmarked deletion & Sannasiddappa et al., 2015 \\
\hline
\end{tabular}

\section{Construction of Gene Mutants}

An allelic replacement mutant of NWMN_0050 and complementation of this mutant were constructed using the previously described method of Horsburgh et al. (2004) using the primers listed in Table 2. Allelic replacement mutants of $\operatorname{tag} \mathrm{O}$ and $\operatorname{arcA}$ in strain Newman were generated by phage transduction (Horsburgh et al., 2001) from previously described mutants (Table 1).

\section{Data Accession Numbers}

The complete genome sequence of S. epidermidis Tü3298 is available at http://www.ebi.ac.uk/ena/data/view/PRJEB11651 (Moran and Horsburgh, 2016). The Illumina sequence read data generated from the RNA-Seq experiments are available from ArrayExpress database ${ }^{1}$ under accession number E-MTAB-4587.

\section{RESULTS AND DISCUSSION}

\section{Comparative Sapienic Acid Resistance}

We hypothesized that differences in sapienic acid resistance might contribute to the higher frequency and persistence of S. epidermidis on healthy human skin relative to $S$. aureus.

\footnotetext{
${ }^{1}$ http://www.ebi.ac.uk/arrayexpress
}

Consistent with our hypothesis, we identified that the mean sapienic acid MIC of S. epidermidis was approximately three times higher than that of $S$. aureus strains $(p=0.023)$ (Figure 1).

Previous studies revealed that $S$. aureus colonizes the skin of atopic dermatitis sufferers, and its colonization frequency inversely correlates with sapienic acid levels (Takigawa et al., 2005). Sapienic acid is not the only factor on atopic skin to be linked with S. aureus colonization, and levels of other skin lipids and antimicrobial peptides are also linked with colonization (Schafer and Kragballe, 1991; Arikawa et al., 2002; Cho et al., 2010). Although sapienic acid is antimicrobial it remains to be demonstrated that within sebum its activity in vivo reduces S. aureus colonization. These in vitro data presented here support a hypothesis that sapienic acid resistance contributes to skin colonization and persistence. The differential survival data are consistent with the increased frequency of staphylococci, not only S. aureus, on skin of atopic dermatitis patients whose skin lipid levels are reduced (Kong et al., 2012; Soares et al., 2013).

While S. epidermidis strains exhibited a greater sapienic acid MIC compared with S. aureus, S. epidermidis growth was inhibited by lower concentrations than skin colonizing corynebacteria. For example, Corynebacterium stratum which colonizes sebaceous niches has up to 10 times greater sapienic acid MIC (Fischer et al., 2012) than the S. epidermidis strains studied here. 
TABLE 2 | Primers used in this study.

\begin{tabular}{|c|c|c|c|}
\hline Gene name & Primer sequences & Efficiency (\%) & Reference \\
\hline rpoB & $\begin{array}{l}\text { F-GCGAACATGCAACGTCAAG } \\
\text { R-GACCTCTGTGCTTAGCTGTAATAGC }\end{array}$ & 97.0 & This study \\
\hline hu & $\begin{array}{l}\text { F-TाTACGTGCAGCACGTTCAC } \\
\text { R-AAAAAGAAGCTGGTTCAGCAGTAG }\end{array}$ & 90.3 & Duquenne et al., 2010 \\
\hline gyrB Тü3298 & $\begin{array}{l}\text { F-AGAAAAGATGGGACGCCCTG } \\
\text { R-CACCATGAAGACCGCCAGAT }\end{array}$ & 96.6 & This study \\
\hline gyrB Newman & $\begin{array}{l}\text { F-ATCGACTTCAGAGAGAGGTTG } \\
\text { R-CCGTTATCCGTTACTITAATCCA }\end{array}$ & 92.9 & Kenny et al., 2009 \\
\hline сарB & $\begin{array}{l}\text { F-GCGATATGCGTAAGCCAACAC } \\
\text { R-GGTACAGGGCCAGCTGTTAG }\end{array}$ & 91.5 & This study \\
\hline pyrP & $\begin{array}{l}\text { F-CGATGTITGGCGCAACAGTA } \\
\text { R-GCTGGTATITGCGCCTTCG }\end{array}$ & 92.5 & This study \\
\hline$c / p B$ & $\begin{array}{l}\text { F-TGGTGCACCTCCAGGTTATG } \\
\text { R-AGAATCCGTAAGACGACCTTCA }\end{array}$ & 99.0 & This study \\
\hline farR & $\begin{array}{l}\text { F-ACGCCAGCTGTGTGGATTAT } \\
\text { R-AACGACTGCGACCTTGATGT }\end{array}$ & 93.3 & This study \\
\hline sasF & $\begin{array}{l}\text { F-TCACTCTGCGATTGAAGGCA } \\
\text { R-TITCCGGTGCCGAATGATCT }\end{array}$ & 95.0 & This study \\
\hline narH & $\begin{array}{l}\text { F-TGGCCTITCCATTGCATCCT } \\
\text { R-TTCAGTGTCGCCAGCAGTTA }\end{array}$ & 93.6 & This study \\
\hline mcrA (gene knockout) & $\begin{array}{l}\text { F1-ACATGAATTCGGAATTGGTTAAGTTCACTC } \\
\text { R1-CCGGTACCAGAACTCATCTAATA CAGAC } \\
\text { F2-ATAACTGCGGCCGCTGTATCACTTAGGTGTATCA } \\
\text { R2-CGACGGATCCTCCAGCTGTTACCAGTCCGA }\end{array}$ & - & This study \\
\hline mcrA (complementation) & $\begin{array}{l}\text { F-TTACGGATCCTTAAGTAACTTCTITCAA } \\
\text { R-TTATAAAGCTTACATCATTTCTGTCCCAG }\end{array}$ & - & This study \\
\hline
\end{tabular}

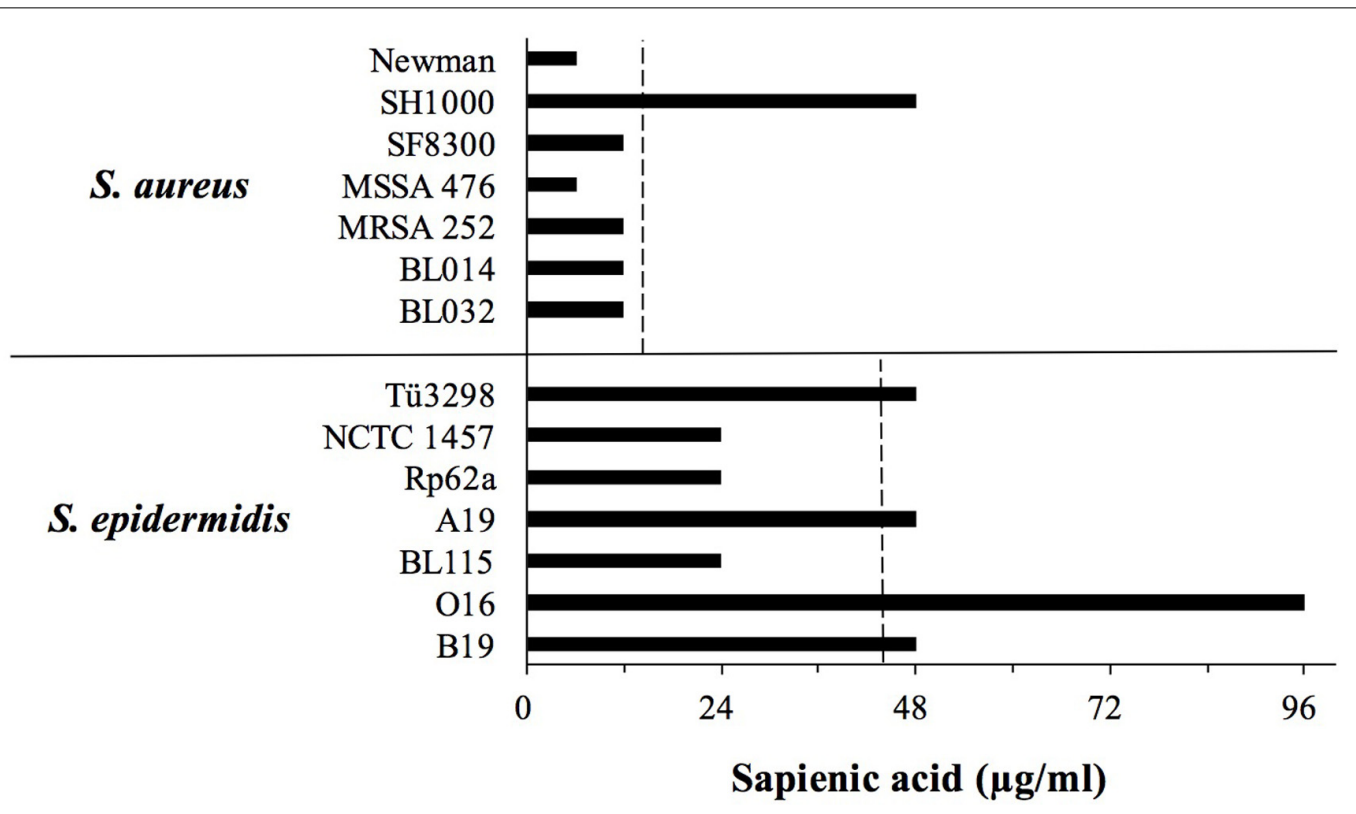

FIGURE 1 | Sapienic acid minimum inhibitory concentration (MICs) of S. aureus and Staphylococcus epidermidis strains. MICs were determined using the broth microdilution method. MICs are the lowest concentration found to inhibit growth. The dotted line shows the mean MIC of each species.

\section{Growth of Sapienic Acid-Challenged S. aureus and S. epidermidis}

Similar to findings of our previous study of the $S$. aureus response to linoleic acid (Kenny et al., 2009), sapienic acid was reported by Neumann et al. (2015) to induce a major adaptive transcriptional response in S. aureus SH1000. We therefore hypothesized that comparison of the transcriptional responses of $S$. aureus with $S$. epidermidis might identify differential skin 
A

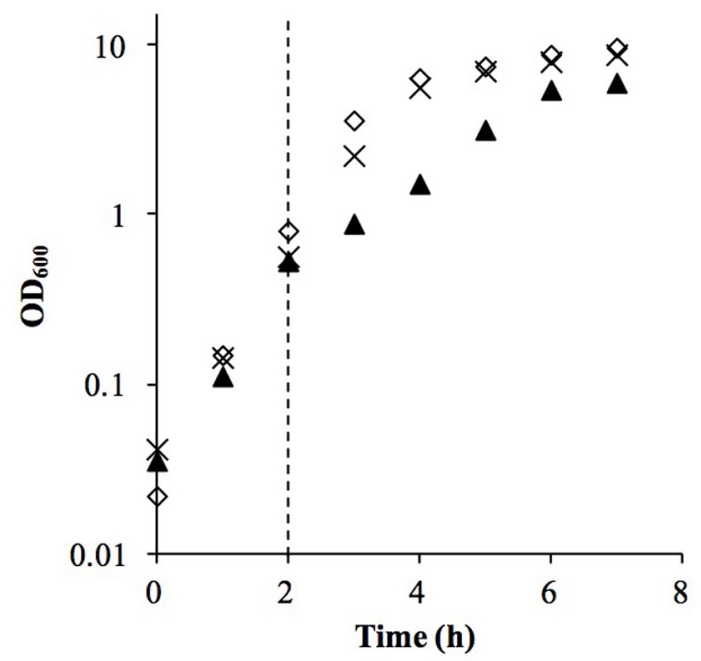

B

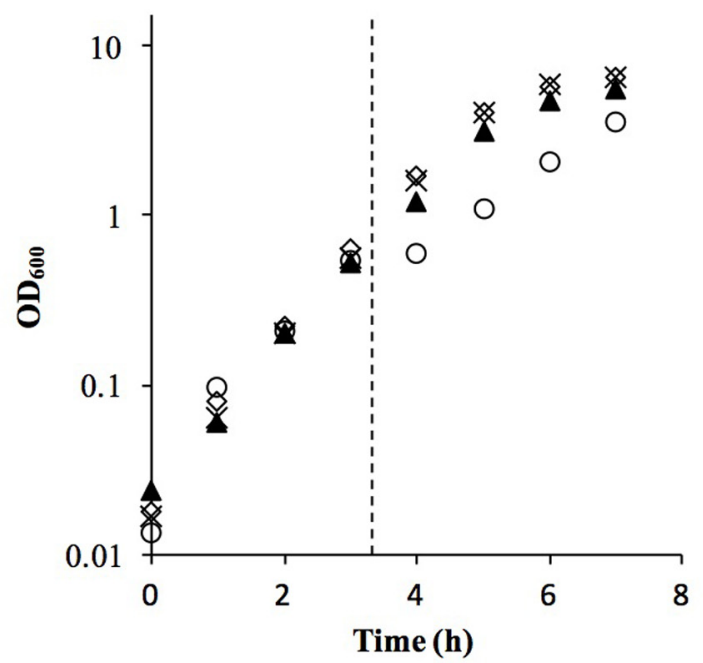

FIGURE 2 | Growth of S. aureus Newman (A) and S. epidermidis Tü3298 (B) challenged with sapienic acid. Bacteria were grown to OD $600=0.5$ before addition of sapienic acid (dotted line). Sapienic acid was added to concentrations $7.5 \mu \mathrm{M}$ (cross), $11.25 \mu \mathrm{M}$ (triangle) and $15 \mu \mathrm{M}$ (circle)-Tü3298 only- or no addition, $0 \mu \mathrm{M}$ (diamond).

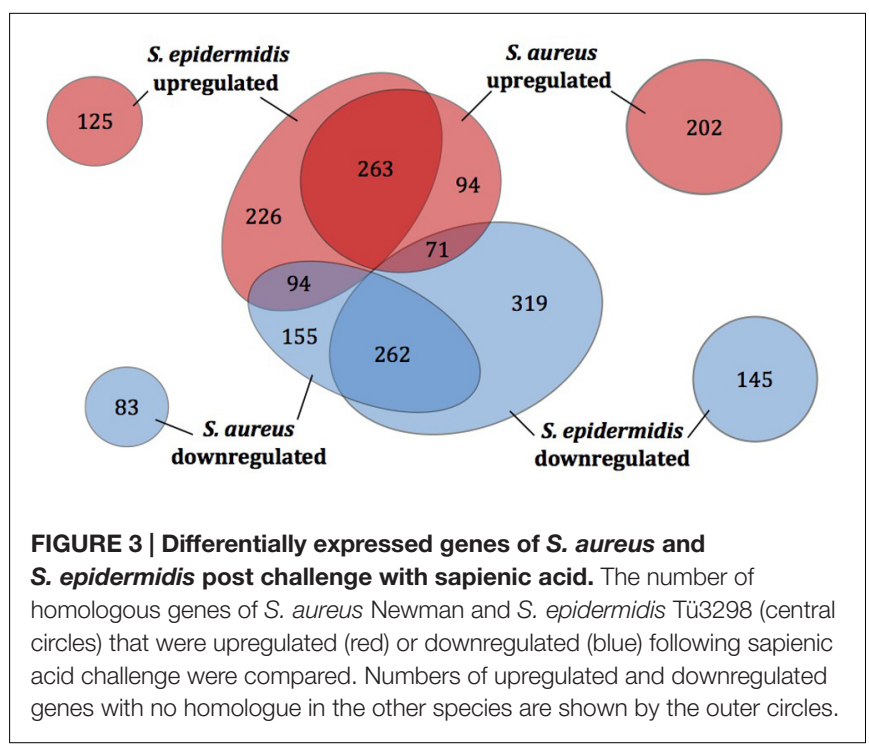

survival mechanisms of these species relating to antimicrobial lipids that might also account for their MIC differences. Since we were interested in the typical response of each species, strains S. aureus Newman and S. epidermidis Tü3298 were selected, being representative of each species based on their MICs.

We used a similar experimental design to our previous linoleic acid transcriptional response study (Kenny et al., 2009), substituting microarrays with RNA-Seq for transcriptomics. Both staphylococci were grown to mid-log phase and challenged with the lowest concentration of sapienic acid that caused an equivalent growth rate reduction (Figure 2), specifically, $11.25 \mu \mathrm{M}$ for S. aureus Newman and $15 \mu \mathrm{M}$ for S. epidermidis
Tü3298. The transcriptomes of each species were determined under challenge and control conditions using RNA-Seq, with resolution of the differentially expressed genes between these conditions.

\section{Sapienic Acid Challenge Transcriptomes}

In response to sapienic acid challenge, $S$. aureus Newman showed 1224 significantly differentially expressed (DE) genes; 630 genes were upregulated and 594 were downregulated (Supplementary Table S1A). S. epidermidis Tü3298 showed 1505 significantly DE genes in response to sapienic acid challenge; 708 genes were upregulated and 797 were downregulated (Supplementary Table $\mathrm{S} 1 \mathrm{~B})$. A greater proportion of $\mathrm{DE}$ genes across the genome was observed for S. epidermidis Tü3298 (64.5\% of 2332 total genes) compared with S. aureus Newman (45.6\% of 2686 total genes).

Based on their homologous gene content, the sapienic acid transcriptomes of $S$. aureus and S. epidermidis were compared, which revealed 525 shared $\mathrm{DE}$ genes with a common regulation pattern (Figure 3, Supplementary Table S1C). Thus, the common transcriptomic response represents less than one half or one third of the DE genes of S. aureus and S. epidermidis, respectively. For both species, a selection of $\mathrm{DE}$ genes were confirmed using $\mathrm{qPCR}$ (Supplementary Figure S1).

Similarities in regulation within the shared responses to sapienic acid are likely to reflect a common response to the membrane depolarization mode of action that disrupts function of the electron transport chain (Cartron et al., 2014). Consistent with this mode of action, we identified upregulated transcription of genes required for sugar uptake, glycolysis, the TCA cycle, NADPH/NADP + recycling and pyruvate metabolism (Figure 4). This shared response may enable the staphylococci to maintain ATP synthesis following disruption of energy generation at the 

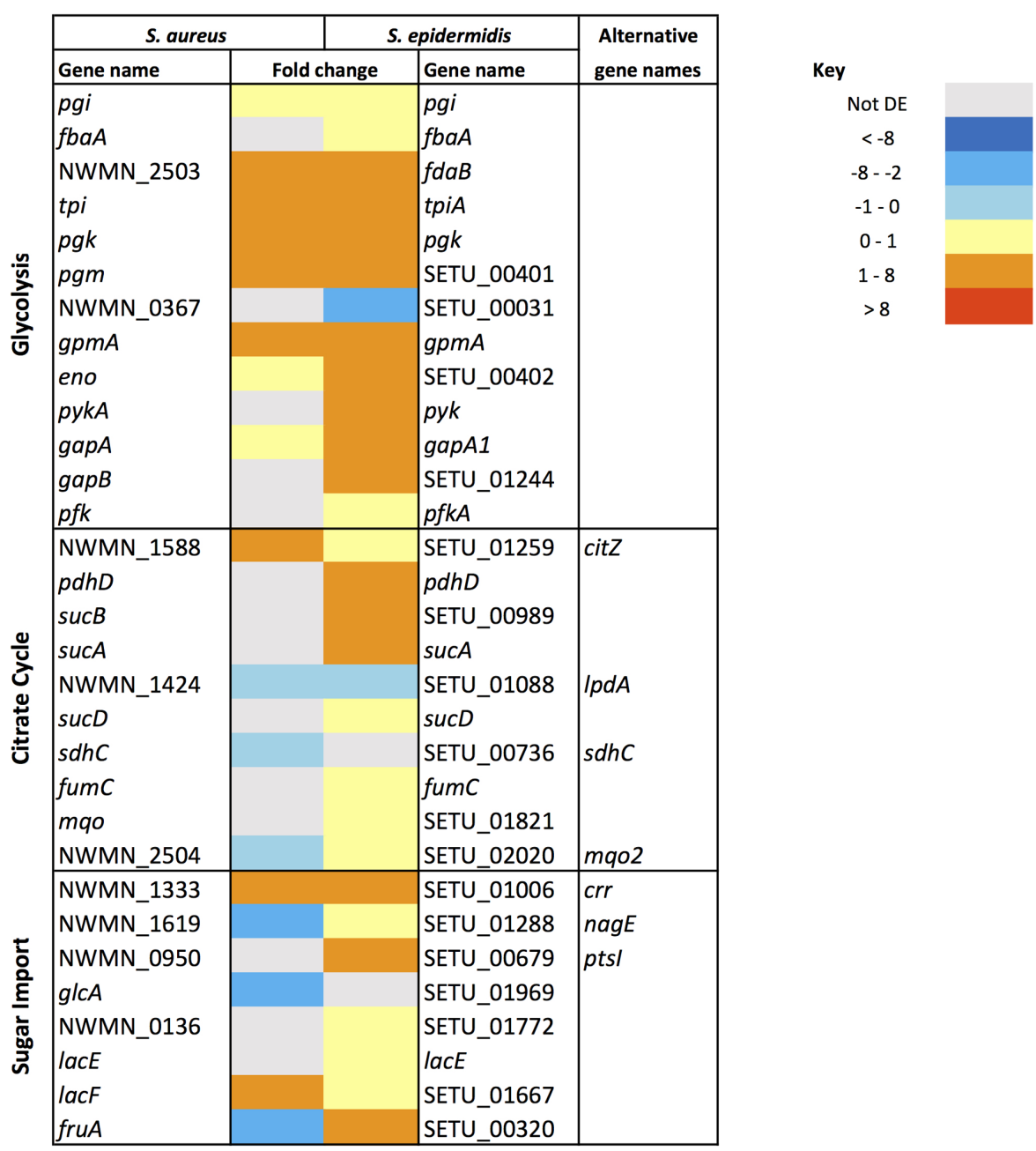

FIGURE 4 | Changes in gene expression of carbon metabolism associated genes in S. aureus and S. epidermidis challenged with sapienic acid.

membrane. Downregulated transcription was associated with genes for cell growth in both species (Figure 5), including peptidoglycan biosynthesis, cell membrane biosynthesis and DNA replication and repair. This response is consistent with adaptation of the bacteria to their changed environment, and with the growth lag observed following sapienic acid challenge of both S. aureus and S. epidermidis (Figure 2).

Downregulation of cell membrane glycerophospholipid biosynthesis genes, when combined with the upregulation of fatty acid degradation genes (aldA, NWMN_1858/SETU_01602, NWMN_2090/SETU_01661, NWMN_2091/SETU_01662), supports the description of sapienic acid incorporation into membrane lipids and lipoproteins in staphylococci (Parsons et al., 2012).

Host fatty acids are metabolized in $S$. aureus by a fatty acid kinase consisting of FakA plus FakB1 or FakB2 subunits in the phospholipid biosynthesis pathway (Parsons et al., 2014). The fakA and fakB1 genes were downregulated in both $S$. aureus Newman and S. epidermidis Tü3298. Gene fakB2 was upregulated only in S. epidermidis; since FakB2 binds long chain unsaturated fatty acids this supports sapienic acid incorporation into the cell phospholipid in this species. Incorporation of AFAs into cellular lipoproteins and phospholipids was suggested as a detoxification mechanism (Desbois and Smith, 2010), while incorporation of fatty acids into lipoproteins enhances the immune response against S. aureus (Nguyen et al., 2015).

\section{Sapienic Acid Transcriptome and Niche Colonization}

The levels of topical skin lipids vary across the body surface (Lampe et al., 1983), and sebaceous richness inversely correlates with staphylococcal frequency (Costello et al., 2009; Coates et al., 2014). Cutaneous skin lipids, including sapienic acid, have potential to act as environmental cues for niche adaptation, particularly with the extensive sapienic aciddependent transcription changes observed that indicate major transcriptome reprofiling in both staphylococci studied here.

Such an environmentally responsive pathway could correspond to the reduced virulence factor expression mediated 


\begin{tabular}{|c|c|c|c|}
\hline \multirow[b]{2}{*}{ Gene name } & \multicolumn{2}{|r|}{ S. epidermidis } & \multirow{2}{*}{$\begin{array}{l}\text { Alternative } \\
\text { gene names }\end{array}$} \\
\hline & $\begin{array}{c}\text { Fold } \\
\text { change }\end{array}$ & Gene name & \\
\hline$m u r B$ & & $m u r B$ & \\
\hline murE & & SETU_00575 & \\
\hline murF & & SETU_01563 & \\
\hline murZ & & - & murA \\
\hline femA & & SETU_00950 & \\
\hline$f m h B$ & & SETU_01718 & femX \\
\hline NWMN_1623 & & SETU_01292 & $s g t A, p b p F$ \\
\hline NWMN_1766 & & SETU_01431 & $s g t B, m g t$ \\
\hline$p b p A$ & & SETU_00752 & $p b p 1$ \\
\hline$p b p 4$ & & SETU_02326 & \\
\hline$p b p C$ & & SETU_01125 & $p b p 3$ \\
\hline$p l s C$ & & SETU_01289 & \\
\hline$p l s X$ & & SETU_00799 & \\
\hline NWMN_1266 & & SETU_00930 & $p l s Y$ \\
\hline $\operatorname{mur} G$ & & $\operatorname{murG}$ & $y p f P$ \\
\hline gpsA & & gpsA & \\
\hline$c d s A$ & & SETU_00830 & \\
\hline $\operatorname{pgs} A$ & & SETU_00852 & \\
\hline NWMN_0164 & & - & \\
\hline NWMN_0984 & & SETU_00717 & \\
\hline NWMN_1614 & & SETU_01283 & \\
\hline$m u t S$ & & SETU_00866 & hexA, mutSI \\
\hline NWMN_1428 & & $x$ seA & \\
\hline recJ & & SETU_01202 & \\
\hline pcrA & & pcrA & \\
\hline NWMN_0358 & & SETU_00026 & $s s b, s s b 1$ \\
\hline NWMN_1909 & & SETU_00604 & $s s b, s s b 2$ \\
\hline$d n a N$ & & dnaN & \\
\hline dnaX & & SETU_00081 & \\
\hline holA & & holA & \\
\hline holB & & SETU_00089 & \\
\hline NWMN_1832 & & SETU_01452 & polC, dnaQ \\
\hline lig & & SETU_01462 & $\operatorname{lig} A$ \\
\hline NWMN_1520 & & SETU_01187 & $\operatorname{rec} D$ \\
\hline ruvA & & ruvA & \\
\hline$r u v B$ & & $\operatorname{ruv} B$ & \\
\hline NWMN_1559 & & SETU_01223 & $\operatorname{tag}$ \\
\hline ung & & SETU_00205 & \\
\hline NWMN_1760 & & SETU_01425 & mut $Y$ \\
\hline NWMN_1460 & & SETU_01130 & nfo \\
\hline
\end{tabular}

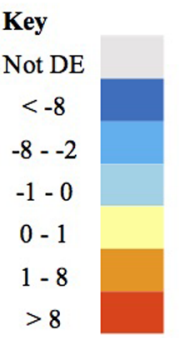




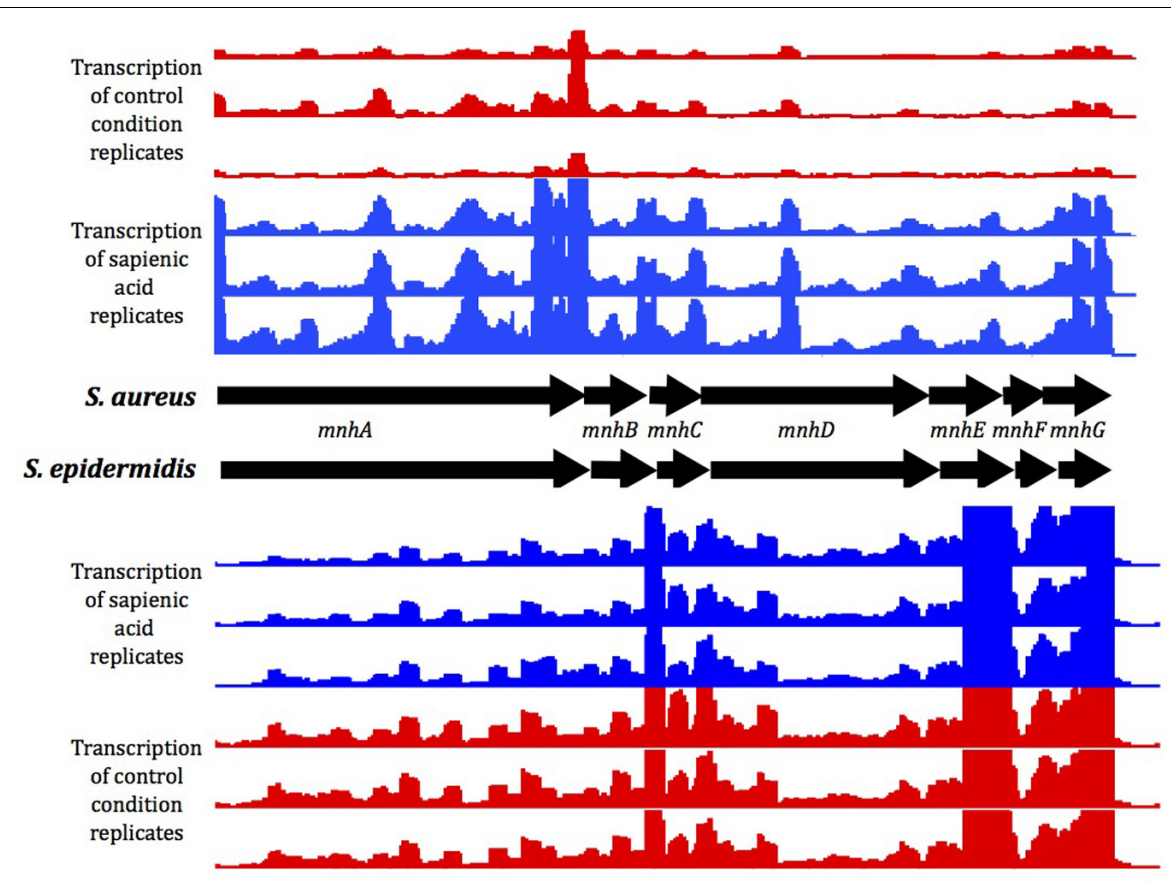

FIGURE 6 | Gene expression of the $\mathbf{m n h}$ operon under control conditions (red) or following sapienic acid challenge (blue). Mapped reads for replicate RNA-Seq data were visualized in integrated genome browser (IGB).

propose that SaeRS is not a key regulator of the sapienic acid survival response of $S$. epidermidis.

Sapienic acid responsive gene expression changes included determinants that protect staphylococci from innate immune defenses of the skin. Adhesin genes implicated in colonization of the nose and skin, such as $s d r C$ in $S$. aureus Newman and ebh genes in S. epidermidis Tü3298, were upregulated (7.4 and 1.3-2.6 fold, respectively) after challenge (Supplementary Table S1). In response to sapienic acid $S$. aureus Newman markedly upregulated (2.8-52.5 fold) capsule biosynthesis genes, approximating the response that we previously reported of $S$. aureus MRSA252 to linoleic acid (Kenny et al., 2009). Despite this pronounced upregulation of capsule biosynthesis genes, Neumann et al. (2015) identified that capsule deficient mutants do not have altered sapienic acid survival, at least for the laboratory strain SH1000 they studied.

Comparing each species' response to sapienic acid there is further distinction between two-component signal transduction systems. The sapienic acid response of S. epidermidis Tü3298 includes upregulation of the $v r a D$ and $v r a E$ which encode an ABC transporter, important for bacitracin resistance. Contrastingly vraDE is not differentially expressed in S. aureus Newman. The VraDE transporter is regulated by the GraRS twocomponent system, that responds to cationic antimicrobial peptides (Pietiainen et al., 2009). Resistance to cationic antimicrobial peptides is an important factor in skin colonization and marks out a further distinction between the responses of each species to sapienic acid, with the potential for a coordinated antimicrobial response in S. epidermidis.

\section{Sapienic Acid Transcriptomes and Resistance}

Comparison of the sapienic acid transcriptomes of S. aureus and $S$. epidermidis revealed candidate genes that might be associated with sapienic acid resistance based upon their upregulation or presence in a pathway. The contribution of these genes to resistance was explored with allelic replacement mutants.

Genes of the mnhABCDEFG operon were all considerably upregulated in $S$. aureus (4.7-6.8 fold change), though less upregulated in S. epidermidis (1.3-1.9 fold change) after sapienic acid challenge. Analysis of transcript abundance data (Figure 6) revealed that expression of the mnh operon was high in S. epidermidis during normal growth (control) conditions (2321341.3 FPKM), while little expression was evident in $S$. aureus in these conditions (22.3-124.1 FPKM). A $m n h F$ in-frame deletion mutant was investigated here for a role in sapienic resistance and had a twofold reduction in MIC $\left(24 \mu \mathrm{g} \mathrm{ml}^{-1}\right)$ compared with isogenic $S$. aureus SH1000 $\left(48 \mu \mathrm{g} \mathrm{ml}^{-1}\right)$. A recent study by Sannasiddappa et al. (2015) determined that $m n h F$ confers resistance to bile salts through efflux of cholic acid. Several mnh genes encode Mrp family secondary antiporter proteins associated with cation/proton transport which can increase the transmembrane electrical potential in staphylococci (Swartz et al., 2007).

In addition to the mnh operon, multiple putative cation antiporters and osmoprotectant transporters were upregulated in response to sapienic challenge in S. aureus (NWMN_2457, NWMN_2050, NWMN_2089 and NWMN_0690) and S. epidermidis (SETU_02263 and SETU_00248-00254). The 
A

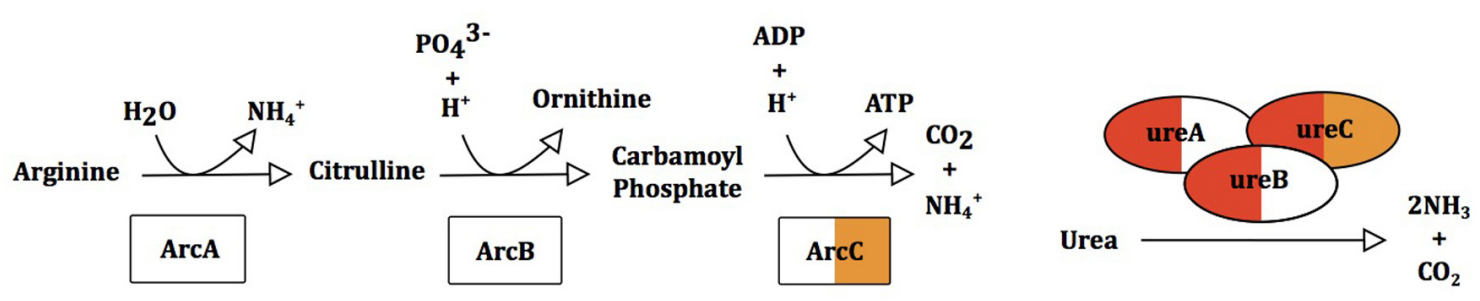

B
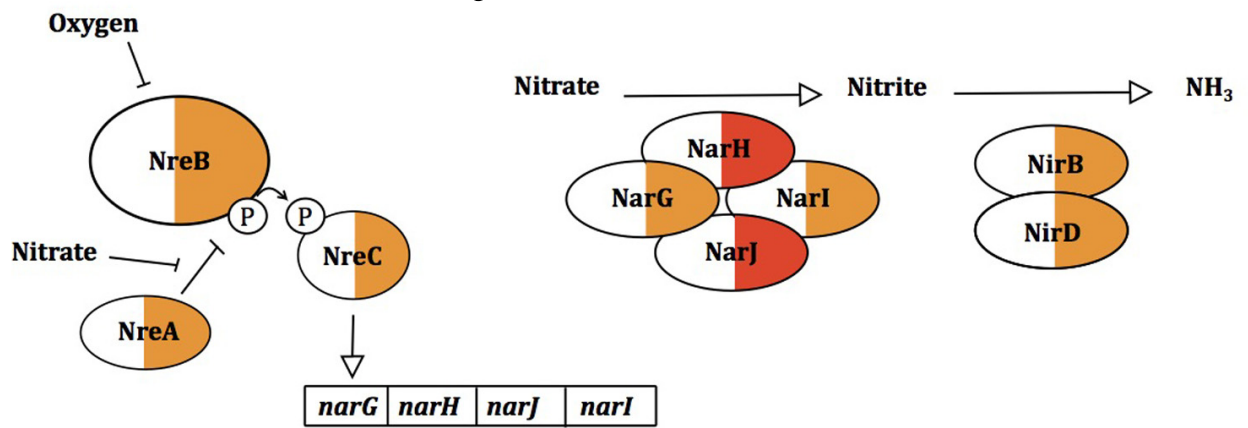

FIGURE 7 | Gene expression changes in ammonia and ammonium producing processes arginine deiminase pathway (A), urease activity (B) and the nitrate reductase pathway (C) in S. aureus and S. epidermidis. Proteins within pathways are colored for S. aureus Newman (left) and S. epidermidis Tü3298 (right) for changes in gene expression following sapienic acid challenge.

transcriptional upregulation of these transporters may protect the cell from the effects of solute leakage and membrane depolarization caused by sapienic acid (Greenway and Dyke, 1979; Parsons et al., 2012).

In response to sapienic acid challenge, S. epidermidis and $S$. aureus upregulate expression of different metabolic pathways that generate ammonia. S. aureus upregulated the urease operon (12.2-13.4 fold) with no consistent differential expression of this operon in S. epidermidis (Figure 7). In contrast, S. epidermidis upregulated $\operatorname{arcC}(1.7$-fold) of the arginine deiminase pathway while the arc operon was not DE in S. aureus. S. epidermidis also upregulated the oxygen-responsive NreABC nitrogen regulation system (1.9-2.5 fold) and likewise upregulated the nitrate and nitrite reduction pathways (1.6-3.6 and 1-2.2 fold, respectively). Nitrate and nitrite dissimilation is coupled to the generation of a proton motive force in anoxic conditions and nitrite dissimilation generates cytoplasmic ammonia (Schlag et al., 2008). The arc, nitrate and nitrite reductase operons are induced in staphylococci only in the absence of oxygen (Fedtke et al., 2002; Lindgren et al., 2014; Nilkens et al., 2014), further supporting that there is reduced uptake or altered perception of oxygen following sapienic acid challenge. S. epidermidis using nitrate as an alternative acceptor for its electron transport chain might offer considerable metabolic flexibility compared with $S$. aureus.

We previously determined that expression of the arginine deiminase pathway operon $(\operatorname{arc} A B C)$ contributes to $S$. aureus linoleic acid resistance (Kenny et al., 2009). The arginine deiminase pathways encoded chromosomally or on the ACME element arc both result in ammonia production, so here we tested if either of these operons contribute to sapienic acid resistance. The sapienic acid MIC was determined for S. aureus SF8300 and

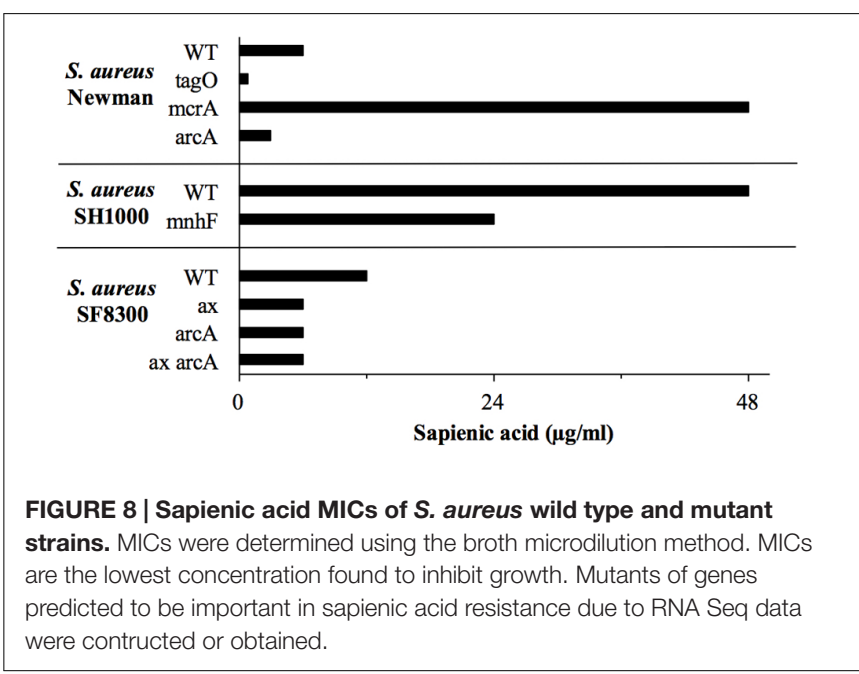

its isogenic ACME element deletion mutant, SF8300ax; the MIC of the mutant was twofold lower than its parent strain (Figure 8). The sapienic acid MIC of the chromosomal $\operatorname{arcA}$ mutants of S. aureus SF8300 and Newman were also twofold lower than their parent strains. While there was no difference in the MIC of SF8300ax and SF8300ax arcA mutant, there was a consistent reduction in growth of the SF8300ax $\operatorname{arcA}$ mutant compared with SF8300ax (at $3 \mu \mathrm{g} / \mathrm{ml}$ sapienic acid in the MIC assay mean $\mathrm{OD}_{600}=0.41$ and 0.94, respectively) (Figure 8).

Staphylococcal myosin cross reactive antigen (McrA) homologs were previously proposed as antimicrobial lipid resistance determinants (Coates et al., 2014) due to their similarity with fatty acid hydratases of streptococci (Bevers et al., 
2009; Volkov et al., 2010; Rosberg-Cody et al., 2011; Joo et al., 2012). Following sapienic acid challenge there was increased expression of the mcrA gene homologs (NWMN_0050 and SETU_00673), 7.4 and 5.4-fold, respectively in S. aureus and $S$. epidermidis. Fatty acid hydratase enzymes convert unsaturated fatty acids into their saturated counterparts, which is a detoxification mechanism for oleic acid in Streptococcus pyogenes (Volkov et al., 2010). The staphylococcal McrA has no obvious secretion motifs making it unlikely to act on extracellular sapienic acid, though it could act to facilitate its metabolism intracellularly. Here, allelic replacement of $m c r A$ in S. aureus was achieved to investigate its contribution to resistance. Somewhat unexpectedly, the sapienic acid MIC of $S$. aureus Newman mcrA (NWMN_0050) was greater $\left(48 \mu \mathrm{g} \mathrm{ml}^{-1}\right)$ than its isogenic parent strain $\left(6 \mu \mathrm{g} \mathrm{ml}^{-1}\right)$ (Figure 8). This increased resistance phenotype was reversed in the Newman mcrA pSK5632+mcrA complementation strain, which had the same MIC as the wild type. Mutation of $m c r A$ takes the survival of $S$. aureus Newman to a level of sapienic acid survival similar to a $S$. epidermidis strain. This indicates that an inability to saturate sapienic acid to palmitic acid through McrA acitivity, or a distinct cellular lipid conversion by McrA impacts $S$. aureus survival. By comparison, the $m c r A$ gene (SETU_006730) of S. epidermidis Tü3298 contains a premature stop codon, indicating this gene activity may not be functional.

Wall teichoic acid (WTA) deficient mutants have reduced MIC for antimicrobial fatty acids (Kohler et al., 2009) and here, $S$. aureus Newman tagO was shown to have a very reduced sapienic acid MIC $\left(<0.8 \mu \mathrm{g} \mathrm{ml}^{-1}\right)$, over eight times lower than its isogenic wild type strain (Figure 8). Despite its importance for survival, genes of the WTA biosynthesis pathway were downregulated in both species $\operatorname{tag} A, \operatorname{tag} G, \operatorname{dlt} X$, and gtaB in $S$. aureus, $\operatorname{tag} B$ and $\operatorname{tag} F / \operatorname{tar} F$ in $S$. epidermidis). In addition, both $S$. epidermidis and $S$. aureus downregulated $d l t, m p r F$, and isdA genes which would be predicted to increase cell hydrophobicity, but these transcription changes may reflect peptidoglycan modification genes mirroring a reduction in cell wall biosynthesis during a period of reduced growth post sapienic acid challenge.

\section{S. epidermidis Specific Resistance Determinants}

Key sapienic acid resistance determinants that differentiate the increased S. epidermidis sapienic acid MIC from $S$. aureus might be identifiable from their signature of transcription upregulation and/or presence only in the S. epidermidis data set. Of those 38 genes upregulated $>2$-fold in S. epidermidis with no homolog in $S$. aureus, 12 are annotated with transport functions (Supplementary Table S2). These genes may counteract the leakage of solutes caused by sapienic acid (Greenway and

\section{REFERENCES}

Allgaier, H., Jung, G., Werner, R., Schneider, U., and Zahner, H. (1986). Epidermin - sequencing of a heterodet tetracyclic 21-peptide amide antibiotic. Eur. J. Biochem. 160, 9-22. doi: 10.1111/j.1432-1033.1986.tb09933.x
Dyke, 1979; Parsons et al., 2012); transport of osmolytes is a key resistance mechanism used by staphylococci during acid stress, where there is also accompanying membrane depolarization (Bore et al., 2007). A further nine genes of the 38 were hypothetical genes, revealing a need to evaluate potential resistance determinants within this gene set.

\section{CONCLUSION}

S. epidermidis strains have greater sapienic resistance than $S$. aureus. The transcriptional responses of $S$. aureus and $S$. epidermidis to sapienic acid reveal that in addition to a shared stimulon, there are multiple distinct pathways modulated in each species. Our data identifies potential roles for the use of alternative respiration pathways, ammonia production and cation/osmolyte transport in differential survival from sapienic acid.

\section{AUTHOR CONTRIBUTIONS}

JM designed and performed experiments and wrote the paper. JA designed and performed experiments. MH conceived and designed experiments and wrote the paper. All authors read and approved submission.

\section{FUNDING}

JM was funded by BBSRC grants BB/D003563/1 and BB/L023040/1 awarded to MH, both with support from Unilever Plc. JA was funded by the Saudi Arabia Ministry of Education. The funders were not involved in the study design, collection of samples, analysis of data, interpretation of data, the writing of this report or the decision to submit this report for publication.

\section{ACKNOWLEDGMENTS}

The Centre for Genomic Research, University of Liverpool, UK carried out rRNA-Seq data generation and processing. We thank Henry F. Chambers, Friedrich Götz, and Simon R. Clarke for sharing strains.

\section{SUPPLEMENTARY MATERIAL}

The Supplementary Material for this article can be found online at: http://journal.frontiersin.org/article/10.3389/fmicb. 2017.00033/full\#supplementary-material

Arikawa, J., Ishibashi, M., Kawashima, M., Takagi, Y., Ichikawa, Y., and Imokawa, G. (2002). Decreased levels of sphingosine, a natural antimicrobial agent, may be associated with vulnerability of the stratum corneum from patients with atopic dermatitis to colonization by Staphylococcus aureus. J. Investig. Dermatol. 119, 433-439. doi: 10.1046/j.1523-1747.2002.01846.x 
Bera, A., Herbert, S., Jakob, A., Vollmer, W., and Gotz, F. (2005). Why are pathogenic staphylococci so lysozyme resistant? The peptidoglycan OM acetyltransferase OatA is the major determinant for lysozyme resistance of Staphylococcus aureus. Mol. Microbiol. 55, 778-787. doi: 10.1111/j.1365-2958. 2004.04446.x

Bevers, L., Pinkse, M., Verhaert, P., and Hagen, W. (2009). Oleate hydratase catalyzes the hydration of a nonactivated carbon-carbon bond. J. Bacteriol. 191, 5010-5012. doi: 10.1128/JB.00306-09

Bieber, T. (2008). Mechanisms of disease: Atopic dermatitis. N. Engl. J. Med. 358, 1483-1494. doi: 10.1056/NEJMra074081

Bore, E., Langsrud, S., Langsrud, O., Rode, T., and Holck, A. (2007). Acidshock responses in Staphylococcus aureus investigated by global gene expression analysis. Microbiology 153, 2289-2303. doi: 10.1099/mic.0.2007/005 942-0

Cartron, M., England, S., Chiriac, A., Josten, M., Turner, R., Rauter, Y., et al. (2014). Analysis of the bactericidal activity of the human skin fatty acid, cis-6hexadecanoic acid on Staphylococcus aureus. Antimicrob. Agents Chemotherapy 58, 3599-3609. doi: 10.1128/AAC.01043-13

Cho, J., Xuan, C., and Miller, L. (2010). Lucky number seven: RNase 7 can prevent Staphylococcus aureus skin colonization. J. Investig. Dermatol. 130, 2703-2706. doi: 10.1038/jid.2010.294

Christensen, G., Simpson, W., Bisno, A., and Beachey, E. (1982). Adherence of slime-producing strains of Staphylococcus epidermidis to smooth surfaces. Infect. Immun. 37, 318-326.

Christensen, G., Simpson, W., Younger, J., Baddour, L., Barrett, F., Melton, D., et al. (1985). Adherence of coagulase negative staphylococci to plastic tissue culture plates: a quantitative model for the adherence of staphylococci to medical devices. J. Clin. Microbiol. 22, 966-1006.

Coates, R., Moran, J., and Horsburgh, M. (2014). Staphylococci: colonizers and pathogens of human skin. Future Microbiol. 9, 75-91. doi: 10.2217/fmb.13.145

Costello, E., Lauber, C., Hamady, M., Fierer, N., Gordon, J., and Knight, R. (2009). Bacterial community variation in human body habitats across space and time. Science 326, 1694-1697. doi: 10.1126/science.1177486

Cue, D., Junecko, J., Lei, M., Blevins, J., Smeltzer, M., and Lee, C. (2015). SaeRSdependent inhibition of biofilm formation in Staphylococcus aureus Newman. PLoS ONE 10:e0123027. doi: 10.1371/journal.pone.0123027

Davis, K., Stewart, J., Crouch, H., Florez, C., and Hospenthal, D. (2004). Methicillin-resistant Staphylococcus aureus (MRSA) nares colonization at hospital admission and its effect on subsequent MRSA infection. Clin. Infect. Dis. 39, 776-782. doi: 10.1086/422997

Desbois, A., and Smith, V. (2010). Antibacterial free fatty acids: activities, mechanisms of action and biotechnological potential. Appl. Microbiol. Biotechnol. 85, 1629-1642. doi: 10.1007/s00253-009-2355-3

Diep, B., Stone, G., Basuino, L., Graber, C., Miller, A., Des Etages, S., et al. (2008). The arginine catabolic mobile element and staphylococcal chromosomal cassette mec linkage: convergence of virulence and resistance in the USA300 clone of methicillin-resistant Staphylococcus aureus. J. Infect. Dis. 197, 1523U35. doi: 10.1086/587907

Duquenne, M., Fleurot, I., Aigle, M., Darrigo, C., Borezée-Durant, E., Derzelle, S., et al. (2010). Tool for quantification of staphylococcal enterotoxin gene expression in cheese. Appl. Environ. Microbiol. 76, 1367-1374. doi: 10.1128/ AEM.01736-09

Duthie, E., and Lorenz, L. (1952). Staphylococcal coagulase: mode of action and antigenicity. J. Gen. Microbiol. 6, 95-107. doi: 10.1099/00221287-6-1-2-95

Fedtke, I., Kamps, A., Krismer, B., and Gotz, F. (2002). The nitrate reductase and nitrite reductase operons and the narT gene of Staphylococcus carnosus are positively controlled by the novel two-component system NreBC. J. Bacteriol. 184, 6624-6634. doi: 10.1128/JB.184.23.6624-6634. 2002

Fischer, C., Drake, D., Dawson, D., Blanchette, D., Brogden, K., and Wertz, P. (2012). Antibacterial activity of sphingoid bases and fatty acids against Gram positive and Gram negative bacteria. Antimicrob. Agents Chemother. 56, 11571161. doi: 10.1128/AAC.05151-11

Greenway, D., and Dyke, K. (1979). Mechanism of the inhibitory action of linoleic acid on the growth of Staphylococcus aureus. J. Gen. Microbiol. 115, 233-245. doi: 10.1099/00221287-115-1-233

Handke, L. D., Rogers, K. L., Olson, M. E., Somerville, G. A., Jerrells, T. J., Rupp, M. E., et al. (2008). Staphylococcus epidermidis saeR is an effector of anaerobic growth and a mediator of acute inflammation. Infect. Immun. 76, 141-152. doi: 10.1128/IAI.00556-07

Higaki, S., Morohashi, M., Yamagishi, T., and Hasegawa, Y. (1999). Comparative study of staphylococci from the skin of atopic dermatitis patients and from healthy subjects. Int. J. Dermatol. 38, 265-269. doi: 10.1046/j.1365-4362.1999. 00686.x

Holden, M., Feil, E., Lindsay, J., Peacock, S., Day, N., Enright, M., et al. (2004). Complete genomes of two clinical Staphylococcus aureus strains: evidence for the rapid evolution of virulence and drug resistance. PNAS 101, 9786-9791. doi: 10.1073/pnas.0402521101

Horsburgh, M., Aish, J., White, I., Shaw, L., Lithgow, J., and Foster, S. (2002). Sigma B modulates virulence determinant expression and stress resistance: characterization of a Functional rsbU strain derived from Staphylococcus aureus 8325-4. J. Bacteriol. 184, 5457-5467. doi: 10.1128/JB.184.19.5457-5467.2002

Horsburgh, M., Wiltshire, M., Crossley, H., Ingham, E., and Foster, S. (2004). PheP, a putative amino acid permease of Staphylococcus aureus, contributes to survival in vivo and during starvation. Infect. Immun. 72, 3073-3076. doi: 10.1128/IAI.72.5.3073-3076.2004

Horsburgh, M. J., Clements, M. O., Crossley, H., Ingham, E., and Foster, S. J. (2001). PerR controls oxidative stress resistance and iron storage proteins and is required for virulence in Staphylococcus aureus. Infect. Immun. 69, 3744-3754. doi: 10.1128/IAI.69.6.3744-3754.2001

Joo, Y., Jeong, K., Yeom, S., Kim, Y., Kim, Y., and Oh, D. (2012). Biochemical characterization and FAD-binding analysis of oleate hydratase from Macrococcus caseolyticus. Biochimie 94, 907-915. doi: 10.1016/j.biochi. 2011.12.011

Kanehisa, M., and Goto, S. (2000). KEGG: Kyoto encyclopedia of genes and genomes. Nucleic Acids Res. 28, 27-30. doi: 10.1093/nar/28.1.27

Kanehisa, M., Goto, S., Sato, Y., Furumichi, M., and Tanabe, M. (2012). KEGG for integration and interpretation of large-scale molecular data sets. Nucleic Acids Res. 40, D109-D114. doi: 10.1093/nar/gkr988

Kelly, J. (2013). Genomic and Metagenomic Analysis of the Skin Microbiota. Ph.D. thesis, University of Liverpool, Liverpool.

Kenny, J., Moran, J., Kolar, S., Ulanov, A., Li, Z., Shaw, L., et al. (2013). Mannitol utilisation is required for protection of Staphylococcus aureus from human skin antimicrobial fatty acids. PLoS ONE 8:e67698. doi: 10.1371/journal.pone. 0067698

Kenny, J., Ward, D., Josefsson, E., Jonsson, I., Hinds, J., Rees, H., et al. (2009). The Staphylococcus aureus response to unsaturated long-chain free fatty acids: survival mechanisms and virulence implications. PLoS ONE 4:e4344. doi: 10. 1371/journal.pone.0004344

Kluytmans, J., and Wertheim, H. (2005). Nasal carriage of Staphylococcus aureus and prevention of nosocomial infections. Infection 33, 3-8. doi: 10.1007/ s15010-005-4012-9

Kluytmans, J., Vanbelkum, A., and Verbrugh, H. (1997). Nasal carriage of Staphylococcus aureus: epidemiology, underlying mechanisms, and associated risks. Clin. Microbiol. Rev. 10, 505-520.

Kohler, T., Weidenmaier, C., and Peschel, A. (2009). Wall teichoic acid protects Staphylococcus aureus against antimicrobial fatty acids from human skin. J. Bacteriol. 191, 4482-4484. doi: 10.1128/JB.00221-09

Kong, H., Oh, J., Deming, C., Conlan, S., Grice, E., and Beatson, M. (2012). Temporal shifts in the skin microbiome associated with disease flares and treatment in children with atopic dermatitis. Genome Res. 22, 850-859. doi: 10.1101/gr.131029.111

Kreiswirth, B., Lofdahl, S., Betley, M., Oreilly, M., Schlievert, P., Bergdoll, M., et al. (1983). The toxic shock syndrome exotoxin structural gene is not detectably transmitted by a prophage. Nature 305, 709-712. doi: 10.1038/305709a0

Lampe, M., Burlingame, A., Whitney, J., Williams, M., Brown, B., Roitman, E., et al. (1983). Human stratum corneum lipids- characterization and regional variations. J. Lipid Res. 24, 120-130.

Langmead, B., Trapnell, C., Pop, M., and Salzberg, S. (2009). Ultrafast and memoryefficient alignment of short DNA sequences to the human genome. Genome Biol. 10:R25. doi: 10.1186/gb-2009-10-3-r25

Li, M., Diep, B., Villaruz, A., Braughton, K., Jiang, X., Deleo, F., et al. (2009). Evolution of Virulence in epidemic community-associated methicillin-resistant Staphylococcus aureus. PNAS 106, 5883-5888. doi: 10.1073/pnas.0900743106

Libberton, B. (2011). The Ecology of Staphylococcus aureus. Ph.D. thesis, University of Liverpool, Liverpool. 
Lindgren, J., Thomas, V., Olson, M., Chaudhari, S., Nuxoll, A., Schaeffer, C., et al. (2014). Arginine deiminase in Staphylococcus epidermidis functions to augment biofilm maturation through $\mathrm{pH}$ homeostasis. J. Bacteriol. 196, 2277-2289. doi: 10.1128/JB.00051- 14

Mack, D., Siemssen, N., and Laufs, R. (1992). Parallel induction by glucose of adherence and a polysacharide antigen specific for plastic-adherentStaphylococcus-epidermidis - evidence for functional relation to intercellularadhesion. Infect. Immun. 60, 2048-2057.

Moran, G., Krishnadasan, A., Gorwitz, R., Fosheim, G., Linda, M., Mcdougal, L., et al. (2006). Methicillin-resistant S. aureus infections among patients in the emergency department. N. Engl. J. Med. 355, 666-674. doi: 10.1056/ NEJMoa055356

Moran, J., and Horsburgh, M. (2016). Whole-genome sequence of Staphylococcus epidermidis Tü3298. Genome Announc. 4, e112-e116. doi: 10.1128/genomeA. 00112-16

Moss, B., and Squire, J. (1948). Nose and skin carriage of Staphylococcus aureus in patients receiving penicillin. Lancet 1, 320-325. doi: 10.1016/S0140-6736(48) 92088-1

Nagarajan, V., and Elasri, M. (2007). SAMMD: Staphylococcus aureus microarray meta-database. BMC Genomics 8:351. doi: 10.1186/1471-2164-8-351

Neumann, Y., Ohlsen, K., Donat, S., Engelmann, S., Kusch, H., Albrecht, D., et al. (2015). The effect of skin fatty acids on Staphylococcus aureus. Arch. Microbiol. 197, 245-267. doi: 10.1007/s00203-014-1048-1

Nguyen, M., Hanzelmann, D., Härtner, T., Peschel, A., and Götz, F. (2015). Skinspecific unsaturated fatty acids boost the Staphylococcus aureus innate immune response. Infect. Immun. 84, 205-215. doi: 10.1128/IAI.00822-15

Nilkens, S., Koch-Singenstreu, M., Niemann, V., Gotz, F., Stehle, T., and Unden, G. (2014). Nitrate/oxygen co-sensing by an NreA/NreB sensor complex of Staphylococcus carnosus. Mol. Microbiol. 91, 381-393. doi: 10.1111/mmi. 12464

Nolan, T., Hands, R., and Bustin, S. (2006). Quantification of mRNA using real-time RT-PCR. Nature Protocols 1, 1559-1582. doi: 10.1038/nprot.2006. 236

Parsons, J., Broussard, T., Bose, J., Rosch, J., Jackson, P., Subramanian, C., et al. (2014). Identification of a two-component fatty acid kinase responsible for host fatty acid incorporation by Staphylococcus aureus. Proc. Natl. Acad. Sci. U.S.A. 111, 10532-10537. doi: 10.1073/pnas.1408797111

Parsons, J., Yao, J., Frank, M., Jackson, P., and Rock, C. (2012). Membrane disruption by antimicrobial fatty acids releases low-molecular-weight proteins from Staphylococcus aureus. J. Bacteriol. 194, 5294-5304. doi: 10.1128/JB. 00743-12

Pietiainen, M., Francois, P., Hyyrylainen, H., Tangomo, M., Sass, V., Sahl, H., et al. (2009). Transcriptome analysis of the responses of Staphylococcus aureus to antimicrobial peptides and characterization of the roles of vraDE and vraSR in antimicrobial resistance. BMC Genomics 10:429. doi: 10.1186/1471-216410-429

Robinson, M., Mccarthy, D., and Smyth, G. (2010). EdgeR: a Bioconductor package for differential expression analysis of digital gene expression data. Bioinformatics 26, 139-140. doi: 10.1093/bioinformatics/btp616
Robinson, M., and Oshlack, A. (2010). A scaling normalization method for differential expression analysis of RNA-seq data. Genome Biol. 11, R25. doi: 10.1186/gb-2010-11-3-r25

Rosberg-Cody, E., Liavonchanka, A., Gobel, C., Ross, R., O’sullivan, O., Fitzgerald, G., et al. (2011). Myosin-cross-reactive antigen (MCRA) protein from Bifidobacterium breve is a FAD-dependent fatty acid hydratase which has a function in stress protection. BMC Biochem 12:9. doi: 10.1186/14712091-12-9

Sannasiddappa, T., Hood, G., Hanson, K., Costabile, A., Gibson, G., and Clarke, S. (2015). Staphylococcus aureus MnhF mediates cholate efflux and facilitates survival under human colonic conditions. Infect. Immun. 83, 2350-2357. doi: 10.1128/IAI.00238-15

Schafer, L., and Kragballe, K. (1991). Abnormalities in epidermal lipid-metabolism in patients with Atopic dermatitis. J. Investig. Dermatol. 96, 10-15. doi: 10.1111/ 1523-1747.ep 12514648

Schlag, S., Fuchs, S., Nerz, C., Gaupp, R., Engelmann, S., Liebeke, M., et al. (2008). Characterization of the oxygen-responsive NreABC regulon of Staphylococcus aureus. J. Bacteriol. 190, 7847-7858. doi: 10.1128/JB.00905-08

Soares, J., Lopes, C., Tavaria, F., Delgado, L., and Pintado, M. (2013). A diversity profile from the staphylococcal community on atopic dermatitis skin: a molecular approach. J. Appl. Microbiol. 115, 1411-1419. doi: 10.1111/jam.12296

Swartz, T., Ito, M., Ohira, T., Natsui, S., Hicks, D., and Krulwich, T. (2007). Catalytic properties of Staphylococcus aureus and Bacillus members of the secondary cation/proton antiporter-3 (Mrp) family are revealed by an optimized assay in an Escherichia coli host. J. Bacteriol. 189, 3081-3090. doi: 10.1128/JB.00021-07

Takigawa, H., Nakagawa, H., Kuzukawa, M., Mori, H., and Imokawa, G. (2005). Deficient production of hexadecenoic acid in the skin is associated in part with the vulnerability of atopic dermatitis patients to colonization by Staphylococcus aureus. Dermatology 211, 240-248. doi: 10.1159/000087018

Volkov, A., Liavonchanka, A., Kamneva, O., Fiedler, T., Goebel, C., Kreikemeyer, B., et al. (2010). Myosin cross-reactive antigen of Streptococcus pyogenes M49 encodes a fatty acid double bond hydratase that plays a role in oleic acid detoxification and bacterial virulence. J. Biol. Chem. 285, 10353-10361. doi: 10.1074/jbc.M109.081851

Ye, J., Coulouris, G., Zaretskaya, I., Cutcutache, I., Rozen, S., and Madden, T. (2012). Primer-BLAST: a tool to design target-specific primers for a polymerase chain reaction. BMC Bioinformatics 13:134. doi: 10.1186/1471-2105-13-134

Conflict of Interest Statement: The authors declare that the research was conducted in the absence of any commercial or financial relationships that could be construed as a potential conflict of interest.

Copyright (c) 2017 Moran, Alorabi and Horsburgh. This is an open-access article distributed under the terms of the Creative Commons Attribution License (CC BY). The use, distribution or reproduction in other forums is permitted, provided the original author(s) or licensor are credited and that the original publication in this journal is cited, in accordance with accepted academic practice. No use, distribution or reproduction is permitted which does not comply with these terms. 